\title{
A Case Study of Nanofiltration Using an Irreversible Thermodynamics-Based Model
}

\author{
G. R. Vakili-Nezhaad ${ }^{1,2}$ and Z. Akbari ${ }^{2}$ \\ ${ }^{1}$ Department of Petroleum \& Chemical Engineering, College of Engineering, Sultan Qaboos University, Muscat 123, Oman \\ ${ }^{2}$ Institute for Nanoscience and Nanotechnology, University of Kashan, Kashan 87317-51167, Iran
}

Correspondence should be addressed to G. R. Vakili-Nezhaad, vakili@squ.edu.om

Received 7 December 2011; Accepted 9 January 2012

Academic Editor: Z. Slanina

Copyright (C) 2012 G. R. Vakili-Nezhaad and Z. Akbari. This is an open access article distributed under the Creative Commons Attribution License, which permits unrestricted use, distribution, and reproduction in any medium, provided the original work is properly cited.

\begin{abstract}
The extended Spiegler-Kedem model that can be solved by using genetic algorithm (GA) has been used for study of the rejection of charged and uncharged solutes in a nanofiltration process. In this work, the accuracy and applicability of this model were tested with the data obtained from the nanofiltration of a real solution. The permeated solution was obtained from the whey ultrafiltration system (ultrafiltered whey). The studied process separates lactose and mineral salts from ultrafiltered whey. Moreover, the ultrafiltered whey nanofiltration system has been simulated with higher precision compared to the other sources of existing data.
\end{abstract}

\section{Introduction}

One of the applications of the nanofiltration membranes is the separation of lactose from the whey. Lactic acid is one of the most valuable retentive food material which has a high price. The whey is the main byproduct which is obtained from the cheese production. It contains high concentration of organic matter, mainly proteins and lactose. Study of the mathematical mass transfer modeling of lactose and other ions through the nanofiltration membranes is very important, because applying this modeling in experimental tests can lead to obtaining a product with higher purity. Some researchers have studied the nanofiltration process in situation where ions and uncharged solutes are present in the feed solution. They have predicted amount of solute retention by using the Donnan steric partitioning model (DSPM). Van Der Horst et al. [1] only described salt retention, and not lactose retention, when whey was used as feed. Cuartas-Uribe et al. [2, 3] used the DSPM and the Spiegler-Kedem model (SK) to analyze and predict the retention of only uncharged solutes (lactose) in the nanofiltration process.

The SK model is based on irreversible thermodynamics. This model, at first, was developed for reverse osmosis membranes and for single-solute nonelectrolyte solutions [3]. In this model the membrane is considered as a black box, and it is characterized in terms of solute permeability and the reflection coefficient.

Ahmad et al. [4] proposed extended Spiegler-Kedem (ESK) model by incorporating solute-solute interactions in the nanofiltration of multiple solutes systems. The global equations of ESK model are as follows:

$$
\begin{aligned}
F_{s} & =\exp \left[\frac{-J_{v}\left(1-\sigma_{s}\right)}{P_{\mathrm{ss}}}\left(1+\sum_{i=1}^{n} A_{i}\right)\right], \\
A_{i} & =\frac{P_{\mathrm{si}}\left(C_{\mathrm{pi}}-C_{\mathrm{bi}}\right) \exp \left(J_{v} / k_{i}\right)}{J_{v}\left[C_{\mathrm{ps}}-\left(1-\sigma_{s}\right) C_{s}\right]}, \\
\frac{R_{\mathrm{os}}}{1-R_{\mathrm{os}}} & =\frac{\sigma_{s}\left(1-F_{s}\right)}{1-\sigma_{s}} \exp \frac{-J_{v}}{k_{s}}, \\
J_{v} & =L_{p}\left[\Delta P-\sum_{s=1}^{n} \sigma_{s} a_{s} R_{s} C_{\mathrm{ms}}\right], \\
a_{s} & =\frac{R_{g} T}{m_{s}} .
\end{aligned}
$$


TABLE 1: Analytical characterization of the UF whey [2].

\begin{tabular}{lc}
\hline Solute & Concentration in UF whey $(\mathrm{NF}-\mathrm{Feed}) \mathrm{Kg} / \mathrm{m}^{3}$ \\
\hline $\mathrm{Cl}^{-}$ & 1.29 \\
$\mathrm{Na}^{+}$ & 0.37 \\
$\mathrm{~K}^{+}$ & 1.48 \\
$\mathrm{PO}_{4}{ }^{3-}$ & 0.6 \\
$\mathrm{Mg}^{2+}$ & 0.072 \\
$\mathrm{SO}_{4}{ }^{2-}$ & 0.093 \\
$\mathrm{Lactose}^{2+}$ & 41.9 \\
$\mathrm{Ca}^{2+}$ & 0.289 \\
\hline
\end{tabular}

$J_{v}$ is the total volumetric flux $\left(\mathrm{m}^{3} / \mathrm{m}^{2} \mathrm{~s}\right) . P_{\mathrm{ss}}$ is the solute permeability coefficient of solute $s$ with the consideration of the interaction of solute $s . P_{\mathrm{si}}$ is the solute permeability coefficient of solute $s$ with the consideration of the interaction of solute $i . \sigma_{s}$ is reflection coefficient of solute $s . k_{s}$ is the mass transfer coefficient of solute $s$. $R_{\mathrm{os}}=1-C_{\mathrm{ps}} / C_{\mathrm{bs}}$ is the observed rejection of solute $s$, and $R_{s}=1-C_{\mathrm{ms}} / C_{\mathrm{bs}}$ is true rejection of solute $s$ where $C_{\mathrm{ps}}$ is the permeate concentration of solute $s$. $C_{\mathrm{bs}}$ is the solute concentration in the bulk solution, and $C_{\mathrm{ms}}$ is the solute concentration on the membrane surface $\left(\mathrm{kg} / \mathrm{m}^{3}\right) . L_{p}$ is hydraulic permeability constant of membrane $(\mathrm{m} / \mathrm{Pas}) . \Delta P$ is transmembrane pressure $(\mathrm{Pa})$, and $R_{g}$ is ideal gas constant $\left(8.314 \mathrm{~m}^{3} \mathrm{~Pa} / \mathrm{mol} \mathrm{K}\right) . T$ is the temperature $(\mathrm{K})$. $m_{s}$ is the molar mass of solute $s(\mathrm{~g} / \mathrm{mol})$, and $n$ is the total number of solutes. Vakili-Nezhaad and Akbari [5] have proposed a novel method for solving the ESK model by simulating the multiple solutes nanofiltration systems via genetic algorithm (GA). Their method is independent of the number of solutes and data points (experimental data of observed rejection $\left(R_{o}\right)$ for each solute verses flux $\left(J_{v}\right)$ are taken at different $\Delta P$ s and constant feed rate), and it is able to predict the performance of multiple solutes systems with high precision. In the present work, the performance of UF-whey (ultrafiltered-whey) nanofiltration system was predicted by using the ESK model and GA method. The UFwhey nanofiltration system is a pilot plant equipped with flat and spiral wound membrane modules. The used membranes were Desal 5DL and NF 200. Whey was ultrafiltered to avoid fouling problems in the nanofiltration process [2].

\section{Results and Discussion}

2.1. Feed Characterization. The results of analytical characterization of UF whey are shown in Table 1. It was observed by Cuartas-Uribe et al. that the UF whey did not contain fat and the amount of protein was low [2].

2.2. Membrane Characterization. Cuartas-Uribe et al. used two aromatic polyamide composite membranes. These membranes were NF 200 and Desal 5 DL, supplied by Dow Chemical (USA) and GE Osmonics (USA), respectively [2].

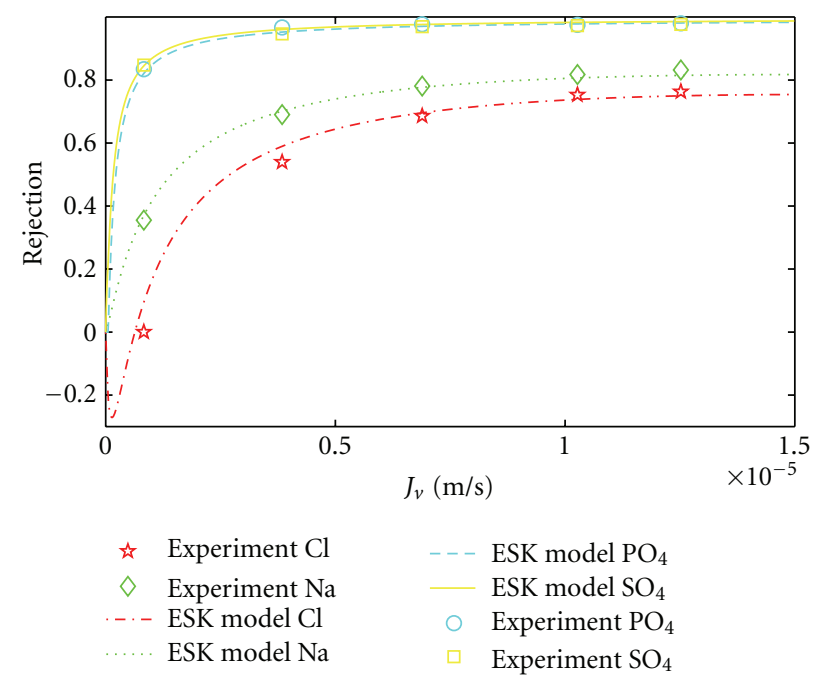

(a)

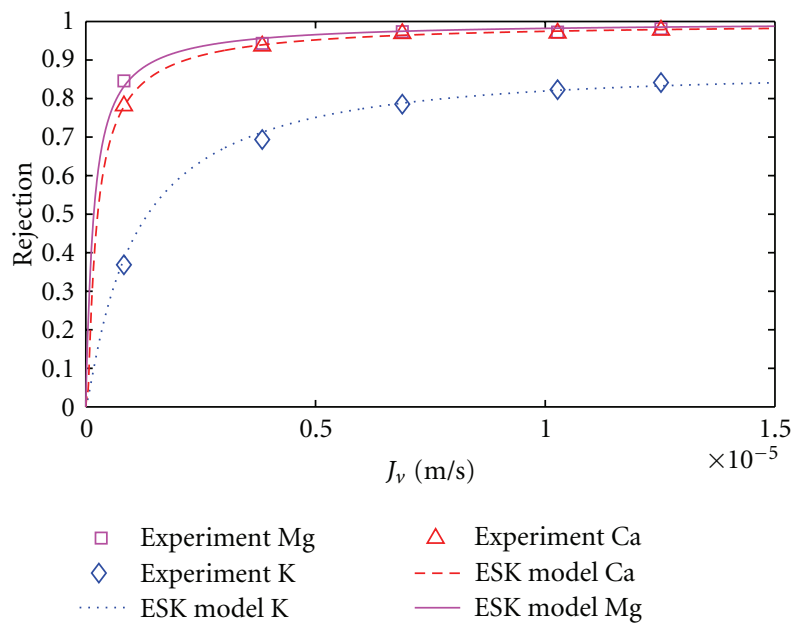

(b)

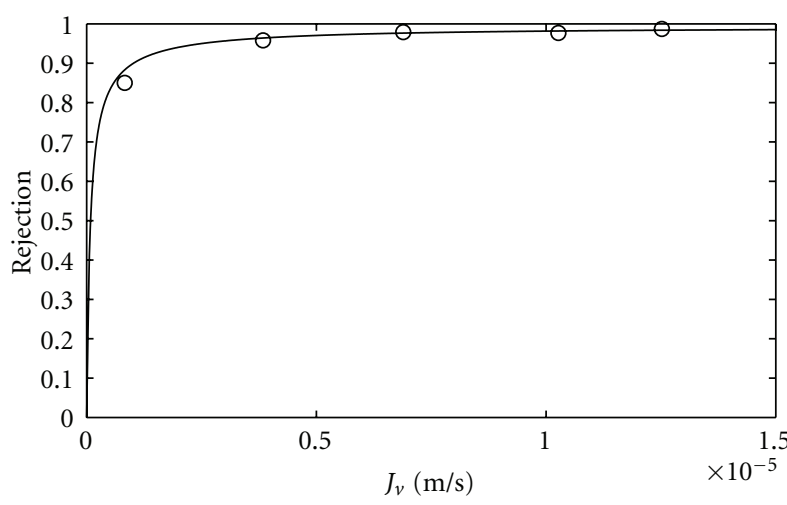

○ Experiment lactose

— ESK model lactose

(c)

FIGURE 1: Ions and lactose rejection of UF whey versus volumetric flux for NF 200 membrane. Experimental results and ESK model by using GA method simulations. (a) $\mathrm{Cl}^{-}, \mathrm{Na}^{+}, \mathrm{PO}_{4}{ }^{3-}$, and $\mathrm{SO}_{4}{ }^{2-}$; (b) $\mathrm{Mg}^{2+}, \mathrm{K}^{+}$, and $\mathrm{Ca}^{2+} ;$ (c) lactose. 
TABLE 2: Parameters estimated from solving ESK model with GA method for NF 200 membrane.

\begin{tabular}{|c|c|c|c|c|c|c|c|c|}
\hline Parameter/s & $\mathrm{Cl}^{-}(1)$ & $\mathrm{Na}^{+}(2)$ & $\mathrm{K}^{+}(3)$ & $\mathrm{PO}_{4}{ }^{3-}(4)$ & $\mathrm{Mg}^{+}(5)$ & $\mathrm{SO}_{4}{ }^{2-}(6)$ & Lactose (7) & $\mathrm{Ca}^{2+}(8)$ \\
\hline$\sigma_{s}$ & 0.8099 & 0.9994 & 0.85978 & 0.9908 & 0.9990 & 0.9950 & 0.9900 & 1.0000 \\
\hline$k_{s}$ & $5.81 e-5$ & $1.51 e-5$ & $9.90 e-4$ & $9.00 e-4$ & $9.00 e-4$ & $9.50 e-4$ & $8.51 e-4$ & $9.50 e-4$ \\
\hline$P_{s 1}$ & $6.86 e-7$ & $9.04 e-11$ & $2.01 e-9$ & $2.00 e-9$ & $3.00 e-10$ & $5.00 e-11$ & $1.01 e-6$ & $8.01 e-9$ \\
\hline$P_{s 2}$ & $1.01 e-7$ & $1.20 e-6$ & $2.01 e-9$ & $2.00 e-9$ & $8.91 e-10$ & $5.01 e-11$ & $2.00 e-9$ & $9.00 e-9$ \\
\hline$P_{s 3}$ & $1.26 e-8$ & $1.00 e-9$ & $9.55 e-7$ & $2.01 e-9$ & $7.61 e-10$ & $5.01 e-11$ & $2.00 e-9$ & $2.00 e-9$ \\
\hline$P_{s 4}$ & $1.27 e-8$ & $1.01 e-9$ & $2.01 e-9$ & $2.07 e-8$ & $5.51 e-10$ & $5.00 e-11$ & $2.01 e-9$ & $2.01 e-9$ \\
\hline$P_{s 5}$ & $3.58 e-6$ & $1.06 e-10$ & $2.00 e-9$ & $2.01 e-9$ & $9.00 e-8$ & $9.00 e-10$ & $2.00 e-9$ & $2.01 e-9$ \\
\hline$P_{s 6}$ & $1.40 e-8$ & $1.03 e-10$ & $2.01 e-9$ & $2.01 e-9$ & $6.90 e-10$ & $7.01 e-8$ & $4.01 e-7$ & $2.01 e-9$ \\
\hline$P_{s 7}$ & $1.20 e-8$ & $5.06 e-10$ & $2.01 e-9$ & $2.01 e-9$ & $1.01 e-10$ & $1.51 e-10$ & $9.99 e-8$ & $1.00 e-9$ \\
\hline$P_{s 8}$ & $1.29 e-8$ & $5.01 e-10$ & $2.01 e-9$ & $2.00 e-9$ & $3.01 e-10$ & $2.51 e-9$ & $2.01 e-8$ & $5.06 e-8$ \\
\hline
\end{tabular}

TABle 3: Parameters estimated from solving ESK model with GA method for Desal 5 DL membrane.

\begin{tabular}{lcccccccc}
\hline Parameter/s & $\mathrm{Cl}^{-}(1)$ & $\mathrm{Na}^{+}(2)$ & $\mathrm{K}^{+}(3)$ & $\mathrm{PO}_{4}{ }^{3-}(4)$ & $\mathrm{Mg}^{+}(5)$ & $\mathrm{SO}_{4}{ }^{2-}(6)$ & $\mathrm{Lactose}(7)$ & $\mathrm{Ca}^{2+}(8)$ \\
\hline$\sigma_{s}$ & 0.999 & 0.6498 & 0.4802 & 0.9043 & 0.8912 & 0.9502 & 0.9428 \\
$k_{s}$ & $5.80 e-6$ & $7.00 e-6$ & $2.00 e-5$ & $5.04 e-5$ & $3.24 e-5$ & $2.50 e-5$ & $8.99 e-4$ & $6.52 e-4$ \\
$P_{s 1}$ & $5.56 e-4$ & $2.00 e-9$ & $2.01 e-9$ & $2.00 e-9$ & $2.99 e-10$ & $5.00 e-11$ & $2.48 e-6$ & $8.01 e-9$ \\
$P_{s 2}$ & $3.14 e-9$ & $3.75 e-6$ & $2.01 e-9$ & $2.00 e-9$ & $8.99 e-8$ & $5.01 e-11$ & $6.52 e-6$ & $9.00 e-9$ \\
$P_{s 3}$ & $1.06 e-8$ & $2.02 e-9$ & $4.06 e-6$ & $2.01 e-9$ & $6.99 e-10$ & $5.01 e-11$ & $5.08 e-7$ & $2.00 e-9$ \\
$P_{s 4}$ & $1.27 e-9$ & $2.00 e-9$ & $1.99 e-9$ & $1.01 e-7$ & $5.50 e-10$ & $5.00 e-11$ & $1.98 e-7$ & $2.01 e-9$ \\
$P_{s 5}$ & $1.58 e-9$ & $1.99 e-9$ & $1.99 e-9$ & $2.01 e-9$ & $1.00 e-7$ & $9.00 e-10$ & $3.00 e-7$ & $2.01 e-9$ \\
$P_{s 6}$ & $3.97 e-10$ & $1.99 e-9$ & $2.07 e-9$ & $2.01 e-9$ & $6.98 e-10$ & $7.01 e-8$ & $7.01 e-8$ & $2.01 e-9$ \\
$P_{s 7}$ & $2.02 e-6$ & $4.99 e-10$ & $1.01 e-9$ & $2.01 e-9$ & $1.41 e-10$ & $7.99 e-11$ & $8.99 e-8$ & $1.00 e-9$ \\
$P_{s 8}$ & $1.29 e-9$ & $2.08 e-10$ & $2.00 e-9$ & $2.00 e-9$ & $3.01 e-10$ & $2.51 e-9$ & $2.01 e-8$ & $1.51 e-7$ \\
\hline
\end{tabular}

\subsection{Results of Solving ESK Model with GA Method}

2.3.1. Genetic Algorithm. In order to find global optimization method, a more powerful method compared to the traditional ones is necessary. In the recent years genetic algorithm (GA) has been found to be an efficient algorithm for the complex engineering calculations. GA is based on the natural selection and the process that drives biological evolution. Those optimization problems that are not well suited for standard optimization algorithms, including problems in which the objective function is discontinuous, nondifferentiable stochastic, or highly nonlinear can be solved by GA. GA differs from a classical, derivative-based, optimization algorithm in two main aspects. First, classical algorithm generates a single point at each iteration and the sequence of points approaches an optimal solution while GA generates a population of points at each generation and the best point in the population approaches to an optimal solution. The second difference is that classical algorithm selects the next point in the sequence by a deterministic computation while GA selects the next population using random number of generators. GA modifies a population of individual solutions between the values of lower and upper bounds of variables successively, in which the initial range only restricts the range of the points in the initial population. At each step, GA selects individuals from the current population stochastically to be the parents of the children for the next generation based on genetic operators including selection, crossover, and mutation. The population size determines the size of population at each generation. Increasing the population size enables GA to search more points, thereby obtaining better results. Fixing the correct population size in a problem is an important factor for proper working of GA. Over a wide range of function optimization problems, a population size of 10 times of the number of variables is recommended. GA moves from generation to generation until the termination criterion is met. The most frequently used stopping criterion is specified maximum number of generations.

2.3.2. Solution of the ESK Model Using GA. The ESK model parameters (solute permeability $\left(P_{\mathrm{ss}}, P_{\mathrm{si}}\right)$, solute reflection coefficient $\left(\sigma_{s}\right)$, and mass transfer coefficient $\left(k_{s}\right)$ ) were obtained by the analysis of real rejection and flux data of Cuartas-Uribe et al. [2,3]. For this purpose the procedure proposed by Vakili-Nezhaad and Akbari [5] was considered (multiobjective optimization method in genetic algorithm by using Matlab 7.6.1 (R2008a), the software package). The results obtained for NF 200 membrane are summarized in Table 2 and for Desal 5DL in Table 3. Figures 1 and 2 compare lactose and ions rejection predicted by the ESK model with the experimental data for two nanofiltration membranes. As can be observed, the results obtained with 


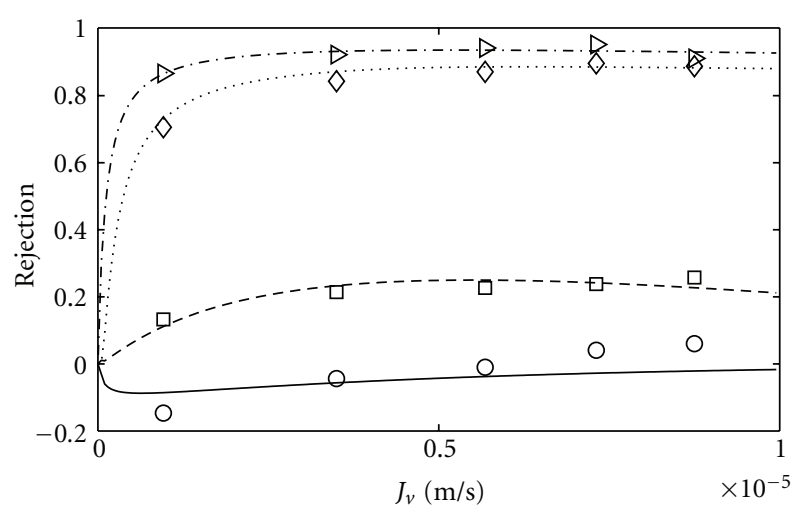

O Experiment $\mathrm{Cl}$ $\square$ Experiment $\mathrm{Na}$ $\diamond$ Experiment $\mathrm{PO}_{4}$ $\triangleright$ Experiment $\mathrm{SO}_{4}$

— ESK model Cl

- - - ESK model Na

-.... ESK model $\mathrm{PO}_{4}$

-..- $\mathrm{ESK}_{\text {model }} \mathrm{SO}_{4}$

(a)

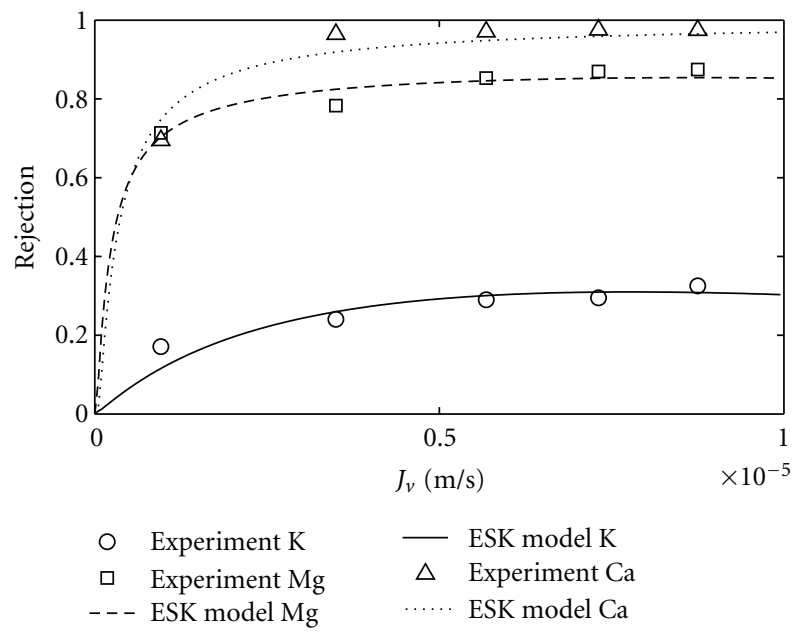

(b)

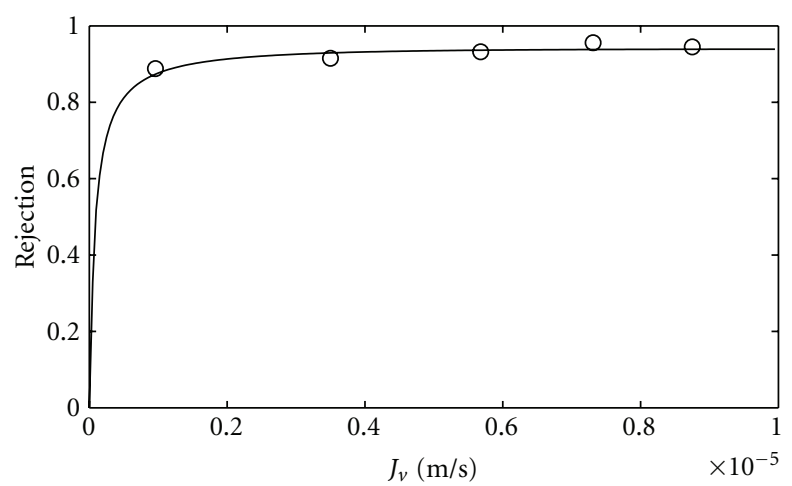

O Experiment lactose

- ESK model lactose

(c)

FIGURE 2: Ions and lactose rejection of UF whey versus volumetric flux for Desal 5 DL membrane. Experimental results and ESK model by using GA method simulations. (a) $\mathrm{Cl}^{-}, \mathrm{Na}^{+}, \mathrm{PO}_{4}{ }^{3-}$, and $\mathrm{SO}_{4}{ }^{2-}$; (b) $\mathrm{Mg}^{2+}, \mathrm{K}^{+}$, and $\mathrm{Ca}^{2+}$; (c) lactose. the ESK model are in a good agreement with the experimental results. In addition, the shape of the curve for lactose and ions rejection versus permeate flux predicted by the ESK model is more similar to the experimental results than the one obtained with the DSPM and SK model by CuartasUribe et al. [3] for two membranes.

\section{Conclusions}

The simulations which were made under the extended Spiegler-Kedem model and were solved by genetic algorithm method are reliable and suitable for predicting the performance of lactose and other solutes in UF-whey nanofiltration system. In addition, the model is able to predict lactose and other ions retention in presence or absence of ions without any information about the membrane structure. In comparison with similar works, this simulation is more perfect and outperforms the precision in the prediction of the performance of membranes in rejection and flux.

\section{References}

[1] H. C. Van Der Horst, J. M. K. Timmer, T. Robbertsen, and J. Leenders, "Use of nanofiltration for concentration and demineralization in the dairy industry: model for mass transport," Journal of Membrane Science, vol. 104, no. 3, pp. 205-218, 1995.

[2] B. Cuartas-Uribe, M. I. Alcaina-Miranda, E. Soriano-Costa, and A. Bes-Piá, "Comparison of the behavior of two nanofiltration membranes for sweet whey demineralization," Journal of Dairy Science, vol. 90, no. 3, pp. 1094-1101, 2007.

[3] B. Cuartas-Uribe, M. C. Vincent-Vela, S. Álvarez-Blanco, M. I. Alcaina-Miranda, and E. Soriano-Costa, "Nanofiltration of sweet whey and prediction of lactose retention as a function of permeate flux using the Kedem-Spiegler and Donnan Steric Partioning models," Separation and Purification Technology, vol. 56, no. 1, pp. 38-46, 2007.

[4] A. L. Ahmad, M. F. Chong, and S. Bhatia, "Mathematical modeling and simulation of the multiple solutes system for nanofiltration process," Journal of Membrane Science, vol. 253, no. 1-2, pp. 103-115, 2005.

[5] G. Vakili-Nezhaad and Z. Akbari, "Modification of the extended Spiegler-kedem model for simulation of multiple solute systems in nanofiltration process," Desalination and Water Treatment, vol. 27, no. 1-3, pp. 189-196, 2011. 

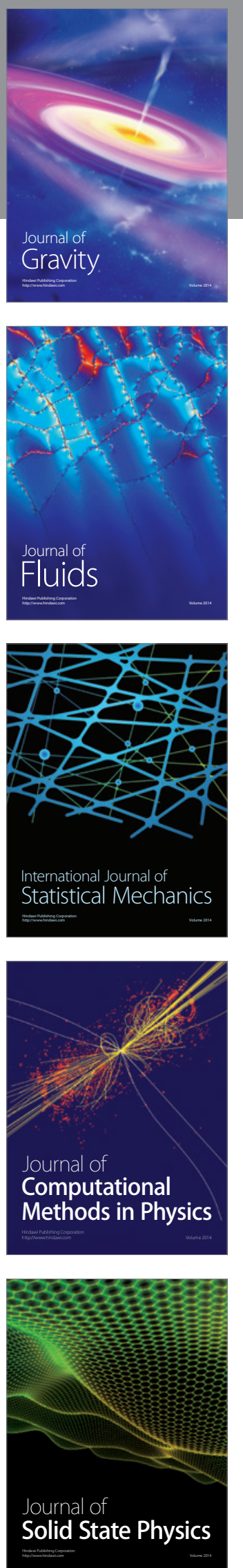
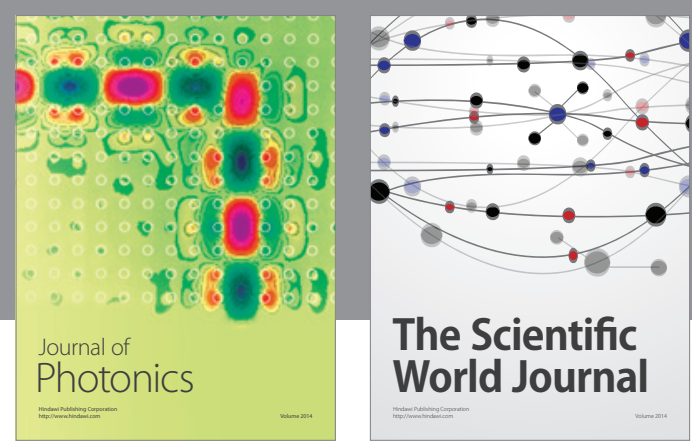

The Scientific World Journal

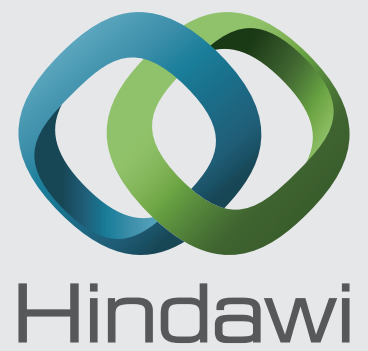

Submit your manuscripts at http://www.hindawi.com
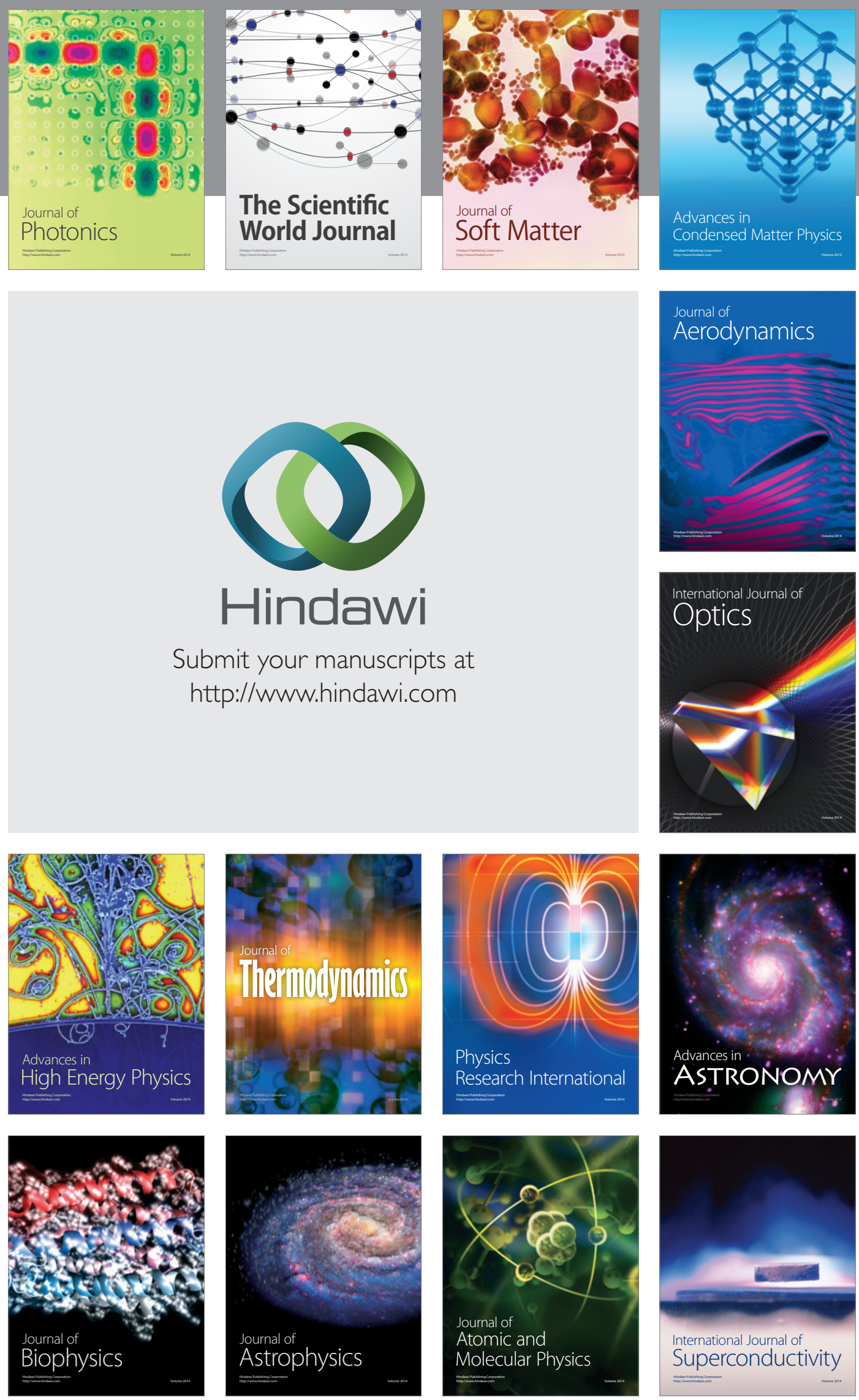
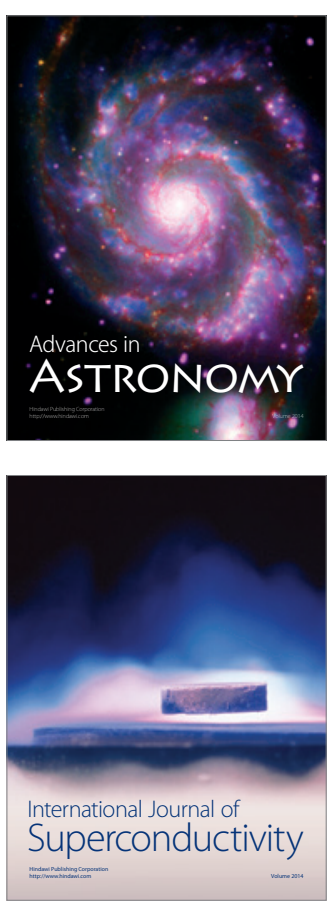